\title{
An Investigation of Attitudes and Information on AIDS
}

Paul O’Mahony ${ }^{*}$

Department of Justice, Dublin

A questionnaire is described which is designed to measure information level on AIDS, defensive attitudes about AIDS, social rejection of AIDS carriers and AIDS sufferers, and understanding of AIDS-related vocabulary. This questionnaire was administered to groups of juvenile offenders $(n=43)$, trainee prison officers $(n=25)$, drug abusers attending a drop-in centre $(n=22)$ and university students $(n=21)$. The level of information about the basic facts of AIDS was found to be encouragingly high in all 4 groups and, complementary - to this, denial of the personal relevance of AIDS and defensive attitudes, which could lead to the avoidance of both communications on AIDS and preventative behavioural change, were found to be very low or non-existent among most subjects in all groups. However, there did appear to be a serious lack of knowledge about the role of sex in the spread of AIDS among a considerable minority of the subjects (up to $20 \%$ in some groups). While socially rejecting attitudes towards AIDS victims were generally absent, they were most common among the offenders and their presence was associated with a poor level of information on AIDS. A poor vocabulary or conceptual understanding regarding AIDS-related concepts was relatively widespread but was not necessarily linked to a lack of basic knowledge about AIDS.

The AIDS epidemic is now a highly threatening, worldwide phenomenon. Although the number of deaths from AIDS in Ireland has been small - Walsh (personal communication, 1988) reported 23 deaths to August, 1988 - there has been an alarming spread of the virus amongst intravenous drug users in Dublin. An official report (Department of Health, 1986) slated that about $30 \%$ of intravenous drug users tested had been found to be HIV seropositive, and it has been estimated that intravenous drug users currently constitute around $60 \%$ of all known sero-positive cases in Ireland (Walsh, 1987). Among other consequences, this has led to special problems for the Irish prison system, because a substantial percentage (about $30 \%$ ) of adults committed to prison are, or have been, intravenous users of drugs (O'Mahony, 1988). In the period up to June, 1988, about 200 offenders have been known HIV carriers, and it is reasonable to assume that the actual figure for HIV carriers passing through the prison system is considerably higher. There is, therefore, an obvious need for remedial measures, especially AIDS education programmes, both within the prison system and throughout society.

Although, according 10 some sources (Loveday, 1987a,b), medical research is making progress towards the discovery of both a vaccine against AIDS and effective curative agents, current scientific opinion (Browning, 1987) holds that both are at least several years away. In the absence of effective medical interventions, the most important weapon against the spread of AIDS is education. Education has the potential to make a very significant contribution in this area because, as far as is known at present, the means by which the virus is spread are restricted to blood-to-blood and semen- or vaginal fluids-to-blood contacts. This suggests that

\footnotetext{
${ }^{*}$ Requests for reprints to Paul O’Mahony, Department of Justice, 15, Lower Hatch Street, Dublin 2.
}

This article is a reproduction of that published in: Irish Journal of Psychology, 10(1) 1989, pp.21-38. Pagination may not match that of the original. 
the public need only 10 be warned against and persuaded to abandon a small number of specific behaviours.

However, the behaviours involved, that is, certain forms of unprotected sexual intimacy (especially sodomy) and the sharing of syringes, raise particular difficulties. An education programme against AIDS must break new ground in an area where social taboo, moral feeling and prejudice against deviancy have formerly held sway. Furthermore, some forms of ignorance are created by embarrassment, bias and myth, rather than by lack of information, and this is especially true in the area of sexual behaviour.

In addition, it is a truism of health education that the relationship between attitude change and behaviour change is by no means direct and predictable. Many self-injurious behaviours, such as cigarette-smoking, are peculiarly resistant to change whatever the level of information and education achieved. A striking AIDS-related example of the failure of information to carry over and achieve change in behaviour was presented by O'Connor \& Stafford-Johnson (1987). They reported that 9 out of 21 HIV positive, pregnant drug addicts attending a Dublin counselling programme, which emphasised the dangers of HIV positive women becoming pregnant, later went on to become pregnant a second time. However, the outlook is, perhaps, generally optimistic in the case of AIDS because programmes are usually aimed at getting people to modify their behaviour rather than abstain totally from established patterns. For example, although total abstinence from drug-taking is highly advisable in its own right, the paramount task of education against AIDS is to persuade the drug user merely to slop sharing syringes. Of course, as Mulleady (1987) pointed out, education is only one term in the equation, and even drug users who are convinced of the need to not share will succumb to sharing if clean syringes are not made widely available.

An important aspect of the work of education against AIDS is the evaluation of information programmes. There are two important stages to this process of evaluation. The first of these is the analysis of the present status of knowledge and information about AIDS held by the relevant target populations. Hastings, Leather \& Scott (1987) have recently argued for the importance of this work, stating that information about AIDS "must be based on a clear understanding of consumer perceptions" so that we can optimise its effectiveness. The second stage is the evaluation of the effectiveness of programmes in improving the knowledge and correcting the misinformation of the target populations.

The present study had two aims. The first was to describe and compare the level of information about AIDS held by four categories of people: recruit prison officers, young offenders, university students and drug abusers. The second was to measure the tendency to deny the personal relevance of AIDS and the tendency to stigmatise carriers of the AIDS virus by the members of these four categories. It was hypothesised that these tendencies, which are of obvious interest in themselves, would be associated with a low level of information about AIDS.

The categories of people chosen to be studied were selected for particular reasons. Recruit prison officers are starting out in a job in which it is highly probable they will meet both known and unknown HIV carriers. They will need to be well informed about possible hazards both for their own sakes and because they may find themselves being looked to by offenders for information and advice. Juvenile offenders in Ireland can be considered a high risk group with respect to HIV, both because they are in, or are about to enter, the age group of highest, and possibly most naive and indiscriminate, sexual activity and because of their relatively high likelihood of exposure to, and involvement in, the intravenous drug using subculture. Similar reasoning would also place university students in the high risk category, although with less certainty. Finally, the drug abusers are, without doubt, the category in Ireland who, at present, face the highest risk of contracting the AIDS virus.

\section{METHOD}

\section{The samples}

Four groups were included in the study: a class of recruit prison officers $(n=25 ; 4$ females and 21 males; mean age 23.7 years); a random sample of male juvenile offenders $(n=43$; 
mean age 18.2 years) from St. Patrick's Institution, a closed detention centre in Dublin for the 16-21 years age group; a class of university students $(n=21 ; 15$ females and 6 males; mean age 22.6 years); and a group of drug users or former users $(n=22 ; 10$ females and 12 males; mean age 26.9 years) attending a drugs counselling centre. Almost all of the drug user group were, or had been, daily or nearly daily intravenous users of opiates. In addition, 7 of the juvenile offenders claimed to have experienced the injection of drugs.

Both the recruit prison officers and the juvenile offenders were tested before being exposed to formal education programmes that were being planned at that time by the Department of Justice, and before the media campaign of the Health Education Bureau. Although the recruits had read a Department of Justice memorandum on AIDS, prepared for Prison Officers, they were tested before receiving lectures on AIDS by a psychologist and a doctor, that are part of the recruit training programme. The students were tested before the Health Education Bureau's media campaign, but the group of drug users was tested after it. It may also be assumed that many of the drug users were exposed to information and advice on AIDS at the counselling centre which they were attending.

The subjects in all four groups were asked to participate in the study, and there was only one refusal-an offender. All subjects who had difficulty with reading were presented with the questionnaire orally, on an individual basis.

\section{The questionnaire}

The questionnaire, which may be seen in the Appendix, was constructed to measure the level of knowledge and understanding of AIDS, and to provide three other measures. Two of these are attitudinal: one concerning the tendency to stigmatise the AIDS victim or HIV carrier, and the other concerning the extent of denial by the individual of the personal relevance and importance of the AIDS issue. The third is a measure of the individual's verbal and conceptual ability in fields in which familiarity is often assumed by media reports on AIDS, such as biology and sexual nomenclature. This latter area is assessed within the questionnaire by five multiple choice questions. The statements in the main body of the questionnaire are judged either true or false, although the respondent may also register 'don't know' to indicate uncertainty, ignorance or confusion on a particular issue.

The multiple choice tests of vocabulary and concept mastery constitute the first part of the questionnaire. In the second part of the questionnaire, five questions relate to stigmatisation $(1,12,18,26$ and 29), twelve relate to denial $(3,6,8,9,11,13,17,20,23,24$, 28 and 31) and fifteen relate to factual information about AIDS (1, 2, 4, 5, 7, 10, 14, 15, 16, 19, 20, 21, 22, 27 and 29). The latter may be divided into two subsections: seven of the items $(1,2,4,7,15,19$ and 21) concern ostensibly clearcut, unequivocal and crucial pieces of factual information ('basic facts about AIDS') about, for example, the fatal nature of AIDS, the major routes of infection and some useful precautions. The remaining eight items involve 'facts' that are not so well established ('additional facts about AIDS'), although they reflect the current stale of scientific opinion; they are, therefore, in some cases, open to reasonable doubt or subjective interpretation.

Thus, the questionnaire produces five separate measures, as follows:

1) of vocabulary and concept mastery (range of scores: 0-5);

2) of information level with respect to basic (crucial, well-established) facts about AIDS (range of scores: 0-7);

3) of information level regarding additional (less clearcut) facts about AIDS (range of scores: 0-8);

4) of denial of the relevance and importance of AIDS (range of scores: 0-12); and

$5)$ of the tendency to stigmatise AIDS victims and HIV carriers (range of scores: 0-5).

The questionnaire also contains two additional items which do not relate to any of the above scales, but which are of intrinsic interest. Question 25 asks if the respondent has changed his or her behaviour since learning about AIDS, and question 30 asks if the respondent has ever engaged in behaviour with a risk of AIDS. 


\section{RESULTS}

In addition to comparing the mean scores of the four groups on the various measures, the results were also analysed in terms of the way the individual questions were answered. Analysis of the separate questions is important because it can uncover specific areas of ignorance and confusion.

\section{Measure 1: Vocabulary and concepts}

A one-way analysis of variance revealed a significant difference between the groups $(F=42.6$; $\mathrm{df}=3,109 ; \mathrm{p} .001)$ on this measure. The range of scores possible was 0 to 5 ; the mean for the recruit group was 3.64, for the students 4.14 , for the drug users 3.64 and for the offenders was 1.70. Sheffe's procedure for testing the difference between group means indicated that the mean of the offenders was significantly lower (p.05) than the means of any of the other three groups, which did not differ significantly among themselves. The offender group, on average, were wrong on at least three of the five questions, so clearly had great difficulty in understanding and defining such terms as 'virus', 'antibodies', 'heterosexual', 'immune system' and 'AIDS antibody positive'. Indeed, while only six (of 68) recruits, students and drug users scored less than 3 on this test, only ten (of 43) offenders scored more than 2; a difference that was highly statistically significant $\left(x^{2}=50.4 ; d f=1 ; p .001\right)$.

The data on the responses to the individual questions may be seen in Table 1 . The offenders had great difficulty in defining a virus, but it is clear that this was a particularly difficult question because it was relatively poorly answered by all four groups. More important was the offenders almost total ignorance of the term 'antibodies', which was, however, generally understood by the other groups. A large majority of the offenders $(70 \%)$ thought that antibodies are a poison produced by the AIDS virus. A relatively high percentage of the drug users and the recruits, as well as the majority of the offenders, did not understand the term 'heterosexual'. A very high proportion of the offenders did not have a satisfactory grasp of what it entails to be an AIDS virus carrier.

Table 1. Percentage of subjects responding correctly to questions on vocabulary and concepts (Measure 1).

\begin{tabular}{lccccc}
\hline & \multicolumn{5}{c}{ Question number and concept to be defined } \\
\cline { 2 - 6 } Group & 1 & 2 & 3 & 4 & 5 \\
\cline { 2 - 6 } & (virus) & (HIV positive) & (heterosexual) & (immune & (antibodies) \\
& & & & 68 & 96 \\
\\
\hline Recruits & 44 & 72 & 33 & 56 & 76 \\
Offenders & 19 & 51 & 86 & 100 & 9 \\
Students & 52 & 90 & 64 & 95 & 86 \\
Drug users & 36 & 82 & 57 & 80 & 57 \\
\hline Total sample & 33 & 69 & & & 52 \\
\hline
\end{tabular}

\section{Measure 2: Basic facts about AIDS}

The average number of correct, incorrect and uncertain responses given by the subjects in each group on Measure 2 may be seen in Table 2. The recruits, students and drug users on average failed to give a correct response to no more than one in seven of the questions; the offenders failed to respond correctly 1.4 times on average. Indeed, the offenders made less errors than the recruits, though they were more frequently uncertain than them: on average they were uncertain on approximately one in seven of the questions. These results indicate that all groups, including the offenders, had a reasonable grasp of the crucial, basic facts of AIDS. The low level of errors is notable because holding false beliefs in this area (e.g., that AIDS is not a filial or incurable disease, or that the use of condoms and not sharing; syringes are ineffective precautions against AIDS) could lead to dire consequences. One-way analysis of variance of the number of correct responses revealed a significant difference among the groups $(F=3.07 ; d f=3,109 ; p .05)$. Scheffe's procedure indicated that only the means of the students and offenders differed significantly (p.05) from one another. However, despite the fact that this difference in the number of correct responses was statistically significant, there was little difference in the number of errors that the two groups made (on average the offenders made only 0.2 more errors than the students). 
Only three individuals, all of them offenders, scored less than four out of seven on this measure, but fifteen (13\%) scored less than five; of this fifteen, nine were offenders. However, the offenders did not differ from the other groups in terms of the frequency with which they scored five or more as opposed to less than five on this measure $\left(x^{2}=2.34 ; \mathrm{df}=1\right)$.

Table 2. Mean number of correct, incorrect and uncertain responses given by subjects in each group on Measure 2 (M2), "Basic facts on AIDS," and Measure 3 (M3), "Additional information about AIDS."

\begin{tabular}{lllllllll}
\hline $\begin{array}{l}\text { Category } \\
\text { of }\end{array}$ & \multicolumn{2}{c}{ Recruits } & \multicolumn{2}{c}{ Offenders } & \multicolumn{2}{c}{ Students } & \multicolumn{2}{c}{ Drug users } \\
\cline { 2 - 9 } response & M2 & M3 & M2 & M3 & M2 & M3 & M2 & M \\
Correct & 6.0 & 5.3 & 5.6 & 4.5 & 6.5 & 5.4 & 6.0 & 5.7 \\
$\begin{array}{l}\text { Incorrect } \\
\text { Uncertain }\end{array}$ & 0.6 & 0.9 & 0.5 & 0.9 & 0.3 & 1.2 & 0.4 & 0.8 \\
\hline
\end{tabular}

Note. Measure 2 items and Measure 3 contained eight items.

The percentage of subjects in each group giving correct, incorrect and uncertain responses to the questions within Measure 2 are presented in Table 3. Although a substantial majority of each group was correct on all seven questions, two questions (numbers 1 and 21), concerning contagion by touch, and the preventative effectiveness of avoiding injection of drugs and the use of condoms, elicited an appreciable number of incorrect responses from the recruit prison officers and the juvenile offenders. Also, there was a high degree of uncertainly about the role of anal intercourse in the spread of HIV among the recruits and offenders, and about the role of straight sex in the offender group. The results indicate that these four issues should be a particular focus of educational programmes, especially ones aimed at juvenile offenders.

Table 3. Percentage of subjects in each group giving correct (C) and incorrect (I) responses to the items in Measure 2.

\begin{tabular}{llllllll}
\hline Category & \multicolumn{2}{c}{ Recruits } & \multicolumn{2}{c}{ Offenders } & \multicolumn{2}{c}{ Students } & \multicolumn{2}{c}{ Drug users } \\
of \\
\cline { 2 - 9 }
\end{tabular}

Note. The balance of responses were in the 'uncertain' category.

Measure 3: Additional information on AIDS

The mean scores for responses on Measure 3 may be seen in Table 2. All groups did less well on Measure 3 than on Measure 2, but this is to be expected given the less certain status of the 'additional information on AIDS'. When the scores for Measures 2 and 3 were combined, considerable variability was revealed in the results for correct responses. This variability was mainly associated with varying levels of uncertainty rather than differences in the average number of incorrect responses, which varied only between 1.2 and 1.5. A one-way analysis of variance revealed a significant difference $(\mathrm{F}=5.83$; $\mathrm{df}=3,104 ; \mathrm{p} .01)$ between the groups on correct response scores. Scheffe's procedure indicated that the mean of the offenders was 
significantly lower than that of either the students or the drug users (p.05), but not significantly different from that of the recruits.

When the scores for measures 2 and 3 were combined, the offenders were found to have made fewer errors than the recruits and the students. None of the four groups made on average more than 1.5 errors on the 15 information questions. A one-way analysis of variance revealed a significant difference $(F=5.83 ; d f=3,109 ; p .01)$ between the groups. Scheffé's procedure indicated

Table 4. Percentage of subjects in each group giving correct (C) and incorrect (I) responses to the items in Measure 3.

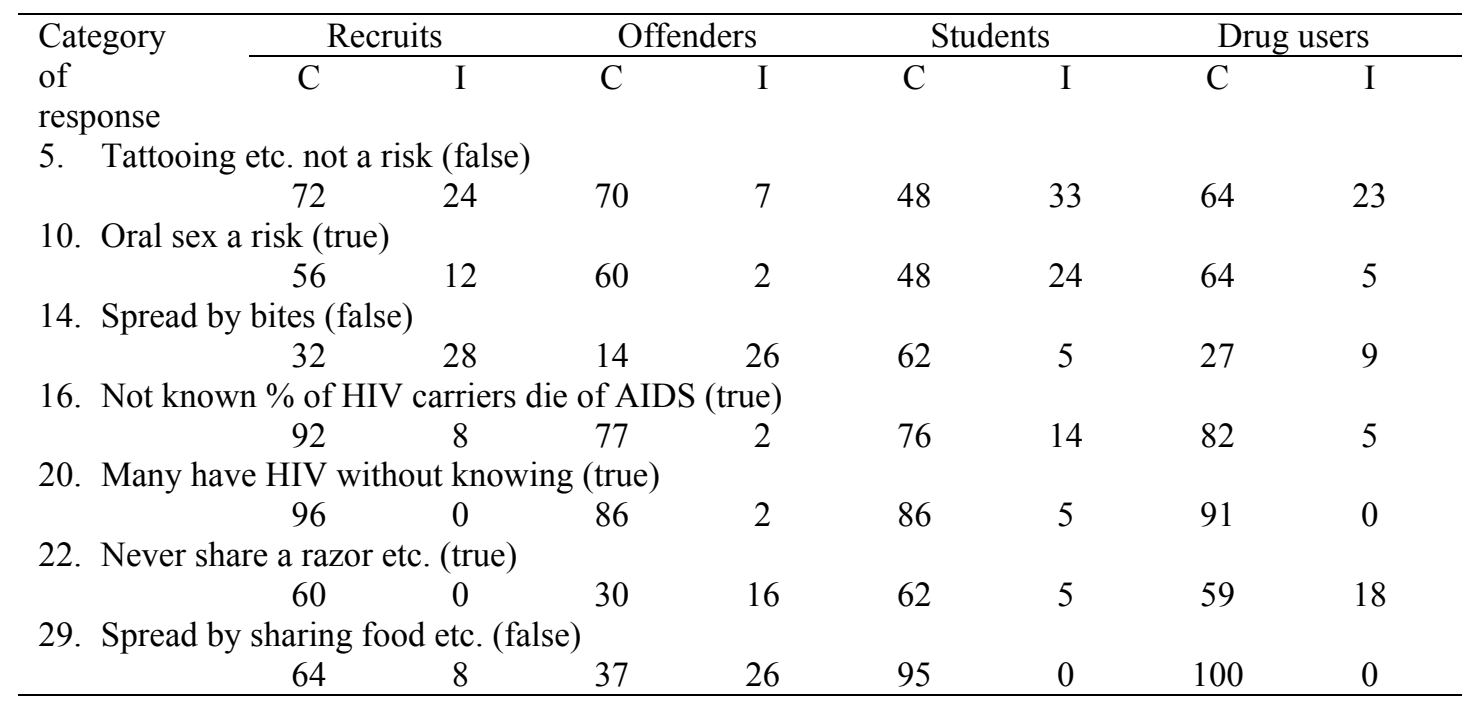

Note. The balance of responses were in the 'uncertain' category.

that the mean of the offenders was significantly lower than that of either the students or the drug users ( $p .05)$, but not significantly different from that of the recruits.

Eight recruits (32\%), four students (19\%), five drug users $(23 \%)$ and twenty-three offenders $(53 \%)$ scored 10 or less correct responses out of the 15 information questions. There was a significant difference between the offenders and the other groups in the frequency with which they scored 10 or less as opposed to more than $10\left(X^{2}=8.30 ; d f=1\right.$; p.01). The most important difference between the offenders and the other groups, however, was in the level of uncertain or confused responses. Only one recruit, one student and none of the drug users answered 5 or more questions in this way, while sixteen $(37 \%)$ of the offenders were uncertain or confused in response to 5 or more of the questions. Indeed, on average the offenders made fewer incorrect responses than both the recruits and the students.

The percentage of subjects in each group giving correct, incorrect and uncertain responses to the questions within Measure 3 are presented in Table 4. There were six instances where a majority of a group failed to give a correct response. In five of these cases the respondents erred on the side of caution; the recruit, offender and drug user groups were not sure that HIV is not spread by bites (question 14) and the offender group were not sure that it is not spread by sneezes, spit etc. (question 27) and by sharing food (question 29). The sixth instance was, by contrast, a failure by the student group to appreciate a possible risk from tattooing, acupuncture or ear-piercing (question 5). On similar lines, only a slight majority of the student group appeared to be aware of the advisability of not sharing razors and toothbrushes (question 22). It is also notable that $34 \%$ of the students, $39 \%$ of the offenders and $44 \%$ of the recruit prison officers were not fully aware that oral intercourse can be a risk for the spread of HIV. These results indicate further areas that deserve to be a particular focus of educational effort.

\section{Measure 4: Denial}

The average scores of all the groups on the denial measure (possible range: $0-12$ ) were very low, indicative of a general absence of denial. The means for the recruits, offenders, students and drug users were $0.76,1.76,1.04$ and 1.72 respectively. Indeed only eleven respondents 
(seven offenders, two students and two drug users) scored above 3 on this measure, and all these scored at a level ( 4 or 5 ) which provides only tentative evidence of denial.

This outcome indicates that the AIDS problem has been taken seriously by all the groups. In general the respondents appeared to regard AIDS as a highly salient issue. Indeed, this awareness and sensitivity to the issue is clearly reflected in the generally good results on the information items of the questionnaire. There was no evidence that large numbers of any of the groups were ignoring sources of information or dismissing the issue of AIDS because they believe, or want to believe that it is of no relevance to themselves.

Measure 5: Stigma The average scores of all the groups on the stigma measure (possible range: 0-5) were also low; the means for the recruits, offenders, students and drug users were $0.56,1.30,0.19$ and 0.23 respectively. A one-way analysis of variance revealed a significant difference $(F=7.85 ; d f=3,109 ;$ p.001) between the groups. Scheffé's procedure indicated that the mean of the offenders was significantly higher than that of cither the students or the drug users (p.05), but not significantly different from that of the recruits, who did not differ significantly from any of the other groups.

The majority of all groups did not tend to stigmatise AIDS victims or HIV carriers. However, if a score of 3 is taken as a cut-off point identifying those holding stigmatising attitudes, a clear difference between the groups is revealed: only one recruit and none of the students or drug users hold a stigmatising view, while ten (almost one quarter) of the offenders do so.

\section{Questions 25 and 30}

Two questions (questions 25 and 30) are of particular interest, although not included in any of the five measures. Question 25 asks if the individual's behaviour has changed since learning about AIDS. The four groups diverge into two sets, which differed significantly $\left(X^{2}=10.20\right.$; $\mathrm{df}=1$; p.01) on this question; thirty $(70 \%)$ of the offenders and seventeen $(77 \%)$ of the drug users, but only eight $(32 \%)$ of the recruits and eight $(38 \%)$ of the students claimed that their behaviour had changed. Question 30 asks if the respondent has ever done anything that has put him/her at risk of catching AIDS. Five $(20 \%)$ of the recruits, thirteen $(30 \%)$ of the offenders, seven $(33 \%)$ of the students, but nineteen $(86 \%)$ of the drug users reported that they had put themselves at risk: a difference between the drug users and the other groups that was statistically significant $\left(X^{2}=20.50 ; \mathrm{df}=1 ; \mathrm{p} .001\right)$.

\section{Intercorrelations between variables}

As the offender group was a particularly homogeneous group of young, single males, the demographic variables of age, sex and marital status were examined only with respect to the other three groups $(N=69)$. Age was significantly correlated with only one of the five measures, but also with the responses to questions 25 and 30. Older respondents did better on Measure $1\left(\mathrm{r}_{\mathrm{s}}=.20 ; p .05\right)$ were more likely to have changed their behaviour $\left(\mathrm{r}_{\mathrm{s}}=.21 ; \mathrm{p} .05\right)$; and were more likely to have been involved in 'at risk' behaviour $\left(r_{s}=.29 ; p .05\right)$. Marital status was significantly related only to responses to question 30 , with married respondents less likely to have been involved in 'at risk' behaviour ( $\mathrm{t}=2.22 ; d f=67 ; \mathrm{p} .05)$. The sex of the respondent was not significantly related to any of the five measures or to the responses to questions 25 and 30.

Measure 1, concerning comprehension of terminology, was positively related to both of the information measures over all 112 respondents (with Measure 2: $r_{s}=.31$; with Measure 3: $\mathrm{r}_{\mathrm{s}}=.33$; ps.001), indicating a slight but significant association between poor vocabulary and poor level of information. However, Measure 1 was negatively related to Measures 4 and 5, denial and stigma (with Measure 4: $r_{s}=-. .28$; with Measure 5: $r_{s}=-.40 ;$ ps.001). This indicates that those with a poor grasp of vocabulary and concepts were more likely to deny the relevance of AIDS and more likely to stigmatise AIDS victims and HIV carriers. Measures 2 and 3, which both concern level of information, also had significant negative correlations with Measures 4 and 5, thus confirming a similar pattern. This set of results then, indicates that relative ignorance with regard to AIDS is associated with both the tendency to minimise the AIDS issue and the tendency to stigmatise AIDS victims and HIV carriers. However, the measures of stigma and denial were not significantly correlated with each other. There was a significant relationship between the responses to questions 25 and $30\left(X^{2}=5.22 ; \mathrm{df}=1 ; \mathrm{p} .05\right)$, 
indicating that those reporting that they had been involved in 'at risk' behaviour were also more likely to report having changed their behaviour. Responses to question 30 were related, slightly but significantly $\left(\mathrm{r}_{\mathrm{s}}=.20 ; p .05\right)$ to the measure of stigma, indicating that those who reported not having ever been involved in 'at risk' behaviour were more likely to stigmatise AIDS victims and HIV carriers.

\section{DISCUSSION}

The most striking feature of the results of this study is the generally high level of information on the basic, central facts of AIDS in all groups, including the juvenile offenders. This latter group had not been exposed to any formal education in this area and, furthermore, contained many young men with only minimal education and often severe literacy problems. Despite these obvious handicaps, the offenders had on the whole an adequate and sensible understanding of the basics of AIDS. These results on information level along with those showing a general absence of denial, indicate that a large majority of the respondents had been exposed to information on AIDS through the media and conversation and were open and attentive to such information that they received.

The present results are consistent with those of Campbell \& Waters (1987) in Britain who found an increased level of public knowledge about AIDS even before Government leafleting and television campaigns. The findings suggest that the response to AIDS is possibly unique in the annals of public health in the extent to which certain rather complex and recherché health matters have rapidly become public knowledge. Health educators, attempting to break through the defenses of the public on such matters as diet, alcohol and cigarette-smoking, might well be envious. On the other hand, levels of information are not good enough to justify complacency. Despite the generally encouraging results, it should be borne in mind that an important minority of individuals, especially juvenile offenders, were shown to have an inadequate grasp of AIDS issues, defined as not knowing 3 of the crucial facts about AIDS. These individuals, comprising about $10 \%$ of all the respondents in this study, are precisely those who need to be specially targeted by educational programmes.

Furthermore, the results also point to certain areas which were widely misunderstood by or problematical for many people, including some with a generally satisfactory information level. Most clearly the evidence suggests that there is widespread doubt about whether the bites, spit, food etc., of an HIV carrier are or are not hazardous. It is uncertain whether any amount of information or validation would dispel these doubts as they probably derive from a scepticism rooted in self-preservation. Misinformation deriving from this kind of scepticism might be termed false positive, since it encourages unwarranted levels of caution. It is the least dangerous form of misinformation from the point of view of containing the AIDS threat. Nonetheless, it requires remedial action, since it is probably related to unnecessary anxiety and also provides a pretext for socially rejecting attitudes towards HIV carriers. On the other hand, the results also suggest that a substantial minority (up to $20 \%$ in some groups) are not clear on the role of anal, oral or vaginal sex in the spread of HIV. These findings, as well as the student group's lack of caution about tattooing, razors, tooth-brushes etc., can be characterized as involving false negative misinformation-misinformation which can facilitate risk-taking behaviour. The logic of prevention dictates that education against AIDS must put a considerable emphasis on combating this false negative misinformation .

Another important finding was the failure of poor performance on the measure of vocabulary and concepts to necessarily predict a poor level of information on AIDS. Despite their generally poor performance on vocabulary and concepts, most offenders were shown to have a satisfactory understanding of the essentials of AIDS. This somewhat unexpected result is encouraging, since it demonstrates that the important basic preventative messages of AIDS education can be successfully conveyed even to those who lack a formal understanding of the underlying phenomena of the disease. On the other hand, there was a small but significant correlation between poor vocabulary and poor level of information. In particular, it is likely that the much greater degree of uncertainty regarding facts about AIDS, displayed by the juvenile offenders, was related to their weakness in terminology. Certainly, the results concerning vocabulary and concepts, which show that a great many respondents, not only offenders, do not fully understand such terms as heterosexual and antibody, strongly suggest that educational messages should be couched in simpler terms or offer simple definitions of difficult terms. 
The results on stigma or social rejection were encouraging, especially in the case of recruits, students and drug users. Only one person (a recruit) from these three groups indicated a markedly rejecting attitude. This positive finding is consistent with Brown et al.'s (1987) discovery of a generally compassionate view towards AIDS victims amongst their large sample of students. However, a considerable proportion (one quarter) of the juvenile offenders displayed a relatively rejecting altitude. It is instructive that the six individuals (including one recruit), who scored very highly (4 or 5) on the measure of stigma, performed poorly on the measure of information on crucial facts of AIDS. This group of 'stigmatizers' had an average score for the crucial facts of AIDS of 3.8, compared with an average of 5.9 for the remaining respondents. Five of these six 'stigmatizers' had a score on the test of crucial information (less than 5) that, it is proposed, indicates that they require further enlightenment on the basic facts of AIDS. These results along with the significant negative correlation between level of information and tendency to stigmatize, strongly suggest a link between ignorance of the facts of AIDS and an inclination to reject AIDS victims and HIV carriers. In this context it is interesting that the evaluators of the Swiss national education programme on AIDS (Lehmann et al., 1987) conclude that their programme succeeded in lowering anxiety and fear of AIDS at the same time as, and probably by the means of, increasing levels of knowledge about AIDS.

The response to questions 25 and 30 was interesting, though hard to interpret. Almost nine out of ten drug users reported that they had been involved in 'at risk' behaviour. This high rate is perhaps not surprising given the prevalence of needle-sharing amongst drug users. However, one in five of the recruit prison officers, who were the group least likely to report 'at risk' behaviour, believed that they had put themselves at risk of AIDS. This is a substantial minority and raises the possibility that this level of fear of having been 'at risk' is related to the kind of false positive information which leads to unwarranted anxiety about AIDS. However, the response to this question was not significantly correlated with level of information about AIDS. It is significant that about one-third of the recruits and the students reported having changed their behaviour since learning about AIDS. Although these are relatively large proportions, far greater numbers of offenders and drug users reported changing their behaviour. It is possible to speculate that these latter groups report more change because they are more likely to have confronted situations that involve 'at risk' behaviour, particularly in the area of drug use.

Finally, the questionnaire has been demonstrated to be a useful research tool for measuring the extent of information and misinformation on AIDS. An important potential use of the questionnaire is for screening, either at the individual level or on a group basis, in order to identify people with a poor understanding of the important issues of AIDS. The questionnaire would also be a useful instrument in the evaluation of the effectiveness of specific AIDS education programmes.

\section{ACKNOWLEDGEMENTS}

I am grateful for the assistance of Fr. Frank Brady, Ana-Liffey Project, Mr. Bill Keaty, Governor, St. Patrick's Institution, Mr. Eamon Kavanagh, Prisons Training Officer, their staffs, Mr. Paul Murphy, Psychologist, and the Prison Officers, offenders, students and drug users who agreed to be interviewed. 